\section{Assessment of autonomic nervous system dysfunction in multiple sclerosis and association with clinical disability}

\author{
Nilufer Kale,' Setty Magana,' \\ Jale Agaoglu, 2,3 Osman Tanik ${ }^{2,3}$ \\ 'Department of Neurology, Mayo Clinic \\ College of Medicine, Rochester, MN, USA; \\ 2Department of Neurology, Okmeydani \\ Training and Research Hospital, Istanbul, \\ Turkey; \\ ${ }^{3}$ Department of Neurology, Apex Medical \\ and Research Center, Istanbul, Turkey
}

\begin{abstract}
Recent studies have reported autonomic dysfunction (AD) in multiple sclerosis (MS), and bladder and/or bowel dysfunction, orthostatic hypotension, and cardiac adaptation disorders have been observed in a wide range of patients $(15-80 \%)$. The primary aim of this study is to investigate the frequency and association of AD in MS patients, assessed by sympathetic skin response (SSR) and a symptoms questionnaire. The secondary aims are to study the association of $\mathrm{AD}$ and disease disability assessed by expanded disability status scale (EDSS), as well as disease duration. One hundred clinically definite MS (CDMS) patients were evaluated for autonomic nervous system (ANS) dysfunction by use of an autonomic symptoms questionnaire and SSR testing. The relationship between these methods, $\mathrm{AD}$ and disease-related parameters, such as the expanded disability status scale (EDSS) and disease duration were all evaluated. Sixtyfive per cent of the patients presented with $\mathrm{AD}$ and $29 \%$ of these patients had abnormal SSR results. MS patients with high EDSS values (EDSS $>4$ ) and longer disease duration were more likely to have ANS dysfunction $(p<0.0001)$. ANS dysfunction is not uncommon in CDMS patients and thus non-invasive investigations of $\mathrm{AD}$ are warranted to optimize $\mathrm{AD}$ evaluation and disease management.
\end{abstract}

\section{Introduction}

Multiple sclerosis (MS) is an inflammatory demyelinating disease of the central nervous system with heterogeneous neurological presentation. Recent studies have reported autonomic dysfunction (AD) in a wide range of patients with MS that might present with abnormalities in the regulation of the cardiovascular system, sleep disturbances and temperature regulation problems $(15-80 \%)$. The pathophysiology behind AD remains under dispute but demyelinating lesions located adjacent to the hypothalamus involving fornix, anterior commissure, internal capsule and optic system might be the basis for autonomic and endocrine malfunction in MS patients. ${ }^{1,2}$ $\mathrm{AD}$ should be considered as one of the crucial components that have an impact on the quality of life outcomes in MS patients and patients need to be questioned for AD. The diagnostic utility of laboratory tests including electrophysiological evaluations for assessing $\mathrm{AD}$ in MS patients has been established and additionally some studies have suggested the use of some self-completed questionnaires on the symptoms of patients with $\mathrm{AD} .^{3-6}$ The primary aim of this study is to investigate the frequency of $\mathrm{AD}$ in MS patients, assessed by sympathetic skin response (SSR) and a symptoms questionnaire, and look for an association between these methods. The secondary aims of this study are to study the association of $\mathrm{AD}$ evaluated by both SSR and symptoms questionnaire, and disability assessed by expanded disability status scale (EDSS), as well as to study the association between $\mathrm{AD}$ and disease duration.

\section{Patients and Methods}

\section{Patient ascertainment}

This prospective study was carried out in the MS Clinic of the Department of Neurology, Okmeydani Training and Research Hospital. After ethical committee approval, one hundred clinically definite MS patients were randomly selected from a larger cohort of patients who were followed in the MS outpatient clinic.? Although McDonald criteria is the latest and commonly accepted diagnostic criteria, we used Poser criteria to establish consistency among the cohort because some patients who were enrolled into the study had long disease duration and Poser criteria had been utilized for their diagnosis. The selection was blinded to clinical complaints relating to autonomic dysfunction to avoid selection bias. In terms of clinical phenotypes, patients with a relapsing remitting disease course or a secondary progressive disease course were enrolled into the study. Patients did not have clinically active disease and did not receive corticosteroid treatment within eight weeks of enrollment. Patients were excluded if comorbidities were present that could interfere with evaluation of autonomic nervous system (ANS) dysfunction (e.g. cardiac disease, alcohol dependency, collagen disease, diabetes mellitus, renal failure,
Correspondence: Nilufer Kale,

200 First St. SW, Rochester, MN, 55905, USA

E-mail: kale.nilufer@mayo.edu

Key words: multiple sclerosis, autonomic nervous system, sympathetic skin response, EDSS, disability.

Acknowledgments: we would like to thank Drs G. Ozdemir and M. Icen.

Contributions: NK manuscript preparation, patient evaluation, patient assessment; SM manuscript preparation; JA patient evaluation, patient assessment; OT manuscript preparation.

Received for publication: 19 January 2009.

Revision received: 11 May 2009.

Accepted for publication: 11 May 2009.

This work is licensed under a Creative Commons Attribution 3.0 License (by-nc 3.0)

(C) Copyright $N$. Kale et al., 2009

Licensee PAGEPress, Italy

Neurology International 2009; 1:e5

doi:10.4081/ni.2009.e5

liver failure, and peripheral neuropathy). Patients were asked to stop anticholinergic medications 48 hours before testing, and drugs such as antidepressants, sympathomimetics, parasympathomimetics, and $B$ blockers were discontinued 24 hours prior to testing. The purpose of the test was fully explained to the patients and informed consent was obtained.

\section{Patient evaluation}

All patients were evaluated by an experienced neurologist by routine neurological examination, including assessment of EDSS. This grading system is considered to be the most practical clinical measure of MS disease activity and is accepted as the primary clinical outcome measure in most MS clinical trials. Because of possible inter-observer variability of EDSS scoring, disability was determined by a single experienced neurologist.

During the neurological evaluation, the same investigator provided patients with a standard questionnaire for the clinical investigation of ANS involvement. Due to the lack of an available validated questionnaire that was translated into Turkish, we developed an inventory of self-reported measures of symptoms relating to autonomic dysfunction based on recent literature. ${ }^{9-13}$ The questionnaire included symptoms of autonomic dysfunction: orthostatic dizziness, palpitations; vasa-secretory motor symptoms (pallor and coldness of skin, increased or lack of sweating in extremities, increased salivation or dryness of mouth or eyes); post-prandial symptoms; bladder problems, bowel problems (constipation); and 
Table 1. Demographic and clinical characteristics of patients.

\begin{tabular}{lc} 
Gender f:m & $2: 1$ \\
Age (years) & $36 \pm 9$ \\
\hline Dd (years) & $7.4 \pm 5.5$ \\
Dc (\%) & $77(77)$ \\
\hline Rrms & $23(23)$ \\
Spms & $3 \pm 1.8$ \\
\hline Edss & $64(64)$ \\
Ans symptoms (\%) & $64(64)$ \\
\hline Bladder problems & $50(50)$ \\
Bowel problems & $27(27)$ \\
\hline Sexual problems & $24(24)$ \\
Cvs problems/orthostatic hypotension & $24(24)$ \\
\hline Thermic problems & $24(24)$ \\
Gastric problems & \\
\hline Values are expressed as mean with standard deviation (sd). Dd; disease duration, dc; disease course, rrms; relapsing remitting ms, \\
spms; secondary progressive ms, edss; expanded disability status scale, ans; autonomic nervous system, cus; cardiovascular system.
\end{tabular}

Table 2. Distribution of autonomic dysfunction among the patient group.

\begin{tabular}{lccc} 
& EDSS $<4(\mathrm{n}=67)$ & $\boldsymbol{p}$ \\
DD & $4.3 \pm 3.1$ & $13.7 \pm 3.5$ & $<0.0001$ \\
SSR abnormality (\%) & $6(9)$ & $23(70)$ & $<0.0001$ \\
\hline Bladder problems (\%) & $33(50)$ & $31(94)$ & $<0.0001$ \\
Bowel problems (\%) & $19(28)$ & $31(94)$ & $<0.0001$ \\
\hline CVS problems (\%) & $2(3)$ & $22(67)$ & $<0.0001$ \\
Sexual problems (\%) & $1(1)$ & $26(79)$ & $<0.0001$ \\
\hline
\end{tabular}

Values are expressed as mean with standard deviation and p value. EDSS; expanded disability status Scale, DD; disease duration, SSR, sympathetic skin reaction,. Group comparisons were evaluated by one-way analysis. Tests applied were two-sided; Wilcoxon's rank test was applied for continuous variables and Kruskal-Wallis rank test was used for ordinal and continuous variables.

sexual problems (erection and ejaculation difficulties, loss of libido/orgasm) (see Appendix). The scoring from the questionnaire was based on answers "yes" (score: 1) and "no" (score: 0) and a total was calculated for each patient. Higher scores indicated more or worse symptoms.

\section{Autonomic nervous system (ANS) assessment, sympathetic skin response}

Patients were instructed to lie down in a comfortable and relaxed position in a quiet, well-lit and air-conditioned room. SSRs were recorded between the hours of 09:00 and 13:00. Five random non-painful electrical stimuli were administered to the median nerve at the wrist $(110 \mathrm{mV}, 0.1 \mathrm{~s})$. Stimuli were applied at irregular intervals, but the delay between two stimuli was always greater than 30 seconds. SSR testing was performed by applying standard surface EMG electrodes (Nihon Kohden EMG) to the palm and the dorsum of the hands and feet with an amplifier gain of 0.1-2 $\mathrm{mV}$ and filter. The amplitude and latency of each response were not assessed as they varied greatly on consecutive stimula- tions. The expected normal response to the electric stimulus is a wave formation with a long duration and low amplitude due to the galvanic sympathetic response because of an instant change in the electric potential of the skin of the palms and feet. When electric stimulus is applied, it is conveyed to the sympathetic centers through the somatosensory pathway (afferent part of the arch). The sympathetic centers, namely the rear hypothalamus and the mesencephalic reticular formation, process the peripheral stimulus and through efferent sympathetic fibers stimulate the sudomotor glands. ${ }^{5,6,14,15}$ SSR is an intersegmental multisynaptic somato-sympathetic reflex and its amplitude varies greatly within and between subjects, making the amplitude or latencies unreliable parameters for the SSR. Moreover, the circadian variability of SSR latencies has been demonstrated in normative studies. Therefore, the result of the test was considered abnormal if no consistent voltage change and wave formation was observed in at least one extremity after the application of electrical stimuli $(100 \mathrm{mV}, 0.1 \mathrm{~s})$. The results were compared with the electrophysiology laboratory normative database results.

\section{Statistical analysis}

Descriptive statistics were applied to the demographic features of the cohort. Group comparisons were evaluated by one-way analysis. Wilcoxon's rank test was applied for continuous variables and Kruskal-Wallis rank test was used for ordinal and continuous variables. Tests were two-sided and a $p<0.05$ was considered statistically significant.

\section{Results}

We identified 100 clinically definite MS patients (68 females, 68\%), the mean age of the patient group was $36 \pm 9$ years. From the patient group, 65 (65\%) patients were assessed to present with autonomic symptoms evaluated by the standard questionnaire (Table 1). Thirtyfive (35\%) patients did not have any autonomic dysfunctions and only 14 (14\%) patients presented with one autonomic dysfunction. SSR evaluation revealed that no wave formation could be elicited in 29 (29\%) patients and was, therefore, considered abnormal. These 29 patients were among the group who had at least one $\mathrm{AD}$ evaluated by the questionnaire and none of the patients without any autonomic symptoms had SSR abnormalities. A statistically significant association was observed between $\mathrm{AD}$ evaluated by the questionnaire and SSR results $(p<0.0001)$. Seventy-seven (77\%) patients presented relapsing-remitting form of MS, and 23 (23\%) patients presented as secondary progressive MS. The mean EDSS score for the patient group was $3 \pm 1.8$. The patients were grouped into 2 categories according to EDSS scores as follows: 1) EDSS < 4.67 (67\%) patients and 2) EDSS >4.33 (33\%) patients. A statistically significant association was observed between EDSS categories and AD results by the questionnaire $(p<0.0001)$. The mean disease duration was $7.4 \pm 5.5$ (range, 1 18 years) and the disease duration was statistically correlated with autonomic impairment $(p<0.0001)$ (Table 2).

\section{Conclusion}

SSR findings seem to reflect axonal conduction blockage due to brain and spinal cord demyelination and, in addition to MS lesions effecting hypothalamus, inflammatory mediators are also considered to have an impact on the control of autonomic and endocrine functioning in MS patients. ${ }^{2}$

Various studies have evaluated the association of disease disability, disease duration and AD evaluated by SS..$^{16-22}$ Our study aims to carry this further with a large sample size and eval- 
uate autonomic system involvement not only by SSR but also by a symptoms questionnaire. Our findings suggest that there is a significant association between ANS dysfunction evaluated by the questionnaire and the electrophysiological evaluation by SSR, as well as disease duration. Disease duration in addition to $\mathrm{AD}$ also most likely correlates with clinical course. EDSS is a grading system widely accepted and used during the follow-up of MS patients to assess the level of disease progression evaluating motor and sensory functions with no emphasis on autonomic involvement. Recent studies including additional autonomic tests have shown that when a multidisciplinary method including evaluation of multiple autonomic system parameters is utilized, there is a higher percentage of $\mathrm{AD}$ abnormality among MS patients. Here we aimed to capture the $\mathrm{AD}$ evaluating a self-assessment method and combining the questionnaire with an available, practical technique. SSR testing has been reported to be an effective method of assessing sympathetic sudomotor outflow disturbances in MS, providing a valuable ancillary method to current electrophysiological procedures. ${ }^{5,14}$ Recent electrophysiological studies found that SSR correlated with the degree of disability evaluated by EDSS and De Seze et al. recently reported that $\mathrm{AD}$ was correlated with spinal cord cross-sectional area reduction but not with spinal cord hyperintensities. ${ }^{5}$ This study confirms that the frequency of $\mathrm{AD}$ in MS, especially in primary progressive forms, has until now been underestimated. Furthermore, AD appears to be more closely related to axonal loss, as demonstrated by spinal cord atrophy, than to demyelinating lesions. In addition, this study found SSR abnormalities in 48\% of progressive MS patients when compared to the relapsing remitting subgroup (30\%). In another study by McDougall et al., no significant association was observed between SSR and MS severity suggesting that cardiovascular and sudomotor autonomic abnormalities in MS patients are likely to be due to plaques distributed throughout the brainstem and spinal cord affecting anatomically widespread autonomic regulatory areas and their connections. ${ }^{16}$

SSR is a beneficial test for sympathetic tract lesions and correlates with the degree of disability and AD in MS patients.,.$^{5,1722}$ Recent studies also described questionnaires that measure autonomic symptoms and presented evidence for validity. ${ }^{9-13}$ This paper also aims to raise the need for a validated ANS dysfunction questionnaire for MS patients and clinicians should be encouraged to use an available questionnaire.

The correlation between SSR testing and a questionnaire suggests that a questionnaire might be an available and simple surrogate tool in assessing autonomic symptoms in clinical trials and epidemiological studies, espe- cially when SSR testing is not available. Autonomic testing seems to be supportive to detect eventual subclinical changes in MS and should be taken into consideration in outcome measures. Further long-term follow-up studies combining clinical, electrophysiological and radiological assessments are warranted to clarify means to evaluate $\mathrm{AD}$, the impacts of multifactorial etiology on MS disability, and to demonstrate the correlation of autonomic dysfunction with other disease-related parameters.

\section{Appendix: questionnaire on clinical autonomic dysfunction}

1. Manifestations occurring after changing position (yes/no): In the past year, have you frequently (at least once a week) experienced one or more symptoms, such as palpitations, dizziness, blurred vision or feeling of weakness, after standing up from a sitting or lying down position?

2. Skin manifestations (at least one abnormal finding) (yes/no): In the past year, have you noticed frequent (at least once a week) changes in skin color, such as red, white or purple? Cutaneous abnormalities at examination?

3. Thermic dysfunctions (at least one abnormal finding) (yes/no): In the past year have you frequently (at least once a week) experienced hot or cold limbs? Thermic modifications found by the examiner? Thermic perception abnormalities comparing hot and cold stimulations?

4. Sphincter dysfunction (at least one abnormal finding) (yes/no): In the past year, have you frequently (at least once a month) lost control of your bladder function? In the past year, have you experienced difficulty in completely emptying your bladder? In the past year, have you frequently (at least once a month) been constipated?

5. Sexual dysfunction (at least one abnormal finding) (yes/no): In the past year, have you frequently (at least once a month) had problems with ejaculation? In the past year, have you experienced difficulty in ejaculation?

6. Gastroparesis (at least one abnormal finding) (yes/no): In the past year, have you frequently (at least once a month) experienced nausea and vomiting after a meal? In the past year, have you experienced feeling full early when eating? In the past year, have you frequently (at least once a month) had abdominal bloating and/or discomfort after a meal? ${ }^{4,6}$

\section{References}

1. Acevedo AR, Nava C, Arriada N, et al. Cardiovascular dysfunction in multiple sclerosis. Acta Neurol Scand 2000;101:858.

2. Huitinga I, De Groot CJ, Van der Valk P, et al. Hypothalamic lesions in multiple sclerosis. J Neuropathol Exp Neurol 2001;60: 1208-18.

3. Flachenecker P, Reiners K, Krauser M, et al. Autonomic dysfunction in multiple sclerosis is related to disease activity and progression of disability. Multiple Sclerosis 2001;7:327-34.

4. Gunal ID, Afsar N, Tanridag T, Aktan S. Autonomic dysfunction in multiple sclerosis: correlation with disease-related parameters. Eur Neurol 2002;48:1-5.

5. De Seze J, Stojkovic T, Gauvrit JY, et al. Autonomic dysfunction in multiple sclerosis: cervical spinal cord atrophy correlates. J Neurol 2001;248:297-303.

6. Nasseri K, Uitdehaag BM, Van Walderveen MA, Ader HJ. Cardiovascular autonomic function in patients with relapsing remitting multiple sclerosis: A new surrogate marker of disease evolution. Eur J Neurol 1999;6:29-33.

7. Mc Donald WI, Compston A, Edan G, et al. Recommended diagnostic criteria for multiple sclerosis: guidelines from the International Panel on the diagnosis of multiple sclerosis. Ann Neurol 2001;50: 121-7.

8. Kurtzke JS. Rating neurological impairment in multiple sclerosis: an Expanded Disability Status Scale (EDSS). Neurology 1983; 33: 1444-52.

9. Suarez GA, Opfer-Gehrking TL, Offord KP, et al. The autonomic symptom profile: a new instrument to assess autonomic symptoms. Neurology 1999;52:523-8.

10. Kröz M, G Feder G, Von Laue HB, et al. Validation of a questionnaire measuring the regulation of autonomic function. BMC Complementary and Alternative Medicine 2008;5:8:2.

11. Mandl T, Granberg V, Jan Apelqvist J, et al. Assessment of autonomic symptoms in diabetics: the Swedish version of the Autonomic Symptom Profile. Clin Physiol Funct Imaging 2008;28;312-7.

12. Seçil Y, Yetimalar Y, Gedizlioglu M, et al. Sexual dysfunction and sympathetic skin response recorded from the genital region in women with multiple sclerosis. Mult Scler 2007;13:742-8.

13. Low PA. Composite autonomic scoring scale for laboratory quantification of generalized autonomic failure. Mayo Clin Proc 1993;68:748-52.

14. Caminero AB, Pérez-Jiménez A, Barreiro 
P, Ferrer T. Sympathetic skin response: correlation with autonomic and somatic involvement in multiple sclerosis. Electromyogr Clin Neurophysiol 1995;35: 457-62.

15. Brazil-Neto JP, Goncalves CA, Araujo CD, Larneiro CR. Circadian latency variability of sympathetic skin response. Clin Auton Res 1998;8:237-9.

16. McDougall AJ, McLeod JG. Autonomic nervous system function in multiple sclerosis. J Neurol Sci 2003;215:79-85.
17. Elie B, Louboutin JP. Sympathetic skin response (SSR) is abnormal in multiple sclerosis. Muscle Nerve 1995;18:185-9.

18. Yokota T, Matsunaga T, Okuyana R. Sympathetic skin response in patients with multiple sclerosis compared with spinal cord transaction and normal controls. Brain 1991;114:1381-94.

19. Drory VE, Nisipeanu PF, Kroczyn AD. Tests of autonomic dysfunction in multiple sclerosis. Acta Neurol Scand 1995;92:356-60.

20. Kodounis A, Stamboulis E, Constantinidis
TS, Liolios A. Measurement of autonomic dysregulation in multiple sclerosis. Acta Neurol Scand 2005;112:403-8.

21. Saari A, Tolonen U, Pääkkö E, et al. Sympathetic skin responses in multiple sclerosis. Acta Neurol Scand 2008;118:22631 .

22. Lorberboym M, Lampl Y, Nikolov G, et al. I123 MIBG cardiac scintigraphy and autonomic test evaluation in multiple sclerosis patients. J Neurol 2008;255:211-6. 